\title{
LXXXIII. A method of finding the coefficients of absorption of the different constituents of a beam of heterogeneous Röntgen rays, or the periods and coefficients of damping of a vibrating dynamical system
}

\section{Sir J.J. Thomson O.M. F.R.S.}

To cite this article: Sir J.J. Thomson O.M. F.R.S. (1915) LXXXIII. A method of finding the coefficients of absorption of the different constituents of a beam of heterogeneous Röntgen rays, or the periods and coefficients of damping of a vibrating dynamical system , Philosophical Magazine Series 6, 30:180, 780-783, DOI: 10.1080/14786441208635457

To link to this article: http://dx.doi.org/10.1080/14786441208635457

\section{9.}

\section{Submit your article to this journal 둔}

\section{Llll Article views: 3}

\section{View related articles 5}


LXXXIII. A Method of Finding the Coefficients of Absorption. of the Different Constituents of a Beam of Heterogeneous Röntgen Rays, or the Periods and Coefficients of Damping of a Vibrating Dynamical System. By Sir J. J.THomson, O.M., F.R.S.*

T HAVE found the following method useful in finding 1 the absorption coefficients of the various constituents of a beam of heterogeneous Röntgen rays. In this case the intensity of the rays after passing through a thickness $d$ of aluminium can be represented by the expression $\mathrm{A}_{1} \epsilon^{-\lambda_{1} d}+\mathrm{A}_{2} \epsilon^{-\lambda_{2} d}+\ldots \mathrm{A}_{n} \varepsilon^{-\lambda_{n}{ }^{d}}$; the problem is to find from the $2 n$ observations which are necessary for this purpose the values of $A_{1}, A_{2}, \ldots A_{n} ; \lambda_{1}, \lambda_{2}, \ldots \lambda_{n}$. Though I arrived at the method from the consideration of the absorption problem, it is of much more general application and applies to any quantity which can be represented as the sum of a series of exponentials of some variable, whether the coefficients of the exponent are real, imaginary, or complex. Since the amplitude of the vibrations, damped or undamped, of a dynamical system about a position of equilibrium can be represented by a series of the form $\sum A \epsilon^{(\alpha+\imath) t}$, the method can be employed to find the periods and damping coefficients of a dynamical system, and is especially useful when we have a graph representing the variation of the displacement of the point in the system with the time. In the case of undamped vibrations it gives a simple method of finding the simple periodic terms into which the motion can be resolved.

The method depends upon the following theorem. Suppose $y_{0}, y_{1}, y_{2}, \ldots y_{2 n-1}$ are $2 n$ observed values, the observations being made at equal intervals $\theta$, of the variable $:$ in the case of the X-ray problem this variable is the thickness of the aluminium leaf through which the radiation has passed; in the dynamical problem the variable is the time. We shall denote this variable by $t$. Then $y_{0}$ is the observation when $t=0, y_{1}$ when $t=\theta, y_{2}$ when $t=2 \theta$, and so on.

Since

$$
\begin{gathered}
y=A_{1} \epsilon^{\lambda_{1} t}+A_{2} \epsilon^{\lambda_{2} t}+A_{3} \epsilon^{\lambda_{3} t}+A_{n} \epsilon^{\lambda_{n} t}, \\
y_{0}=A_{1}+A_{2}+A_{3}+A_{n}, \\
y_{1}=A_{1} \xi+A_{2} \eta+A_{3} \zeta+\ldots, \\
y_{2}=A_{1} \xi^{2}+A_{2} \eta^{2}+A_{3} \zeta^{2}+\ldots, \\
\cdots \cdots \cdots \\
y_{s}=A_{1} \xi^{s}+A_{2} \eta^{s}+A_{3} \xi^{s}+\ldots, \\
\text { * Communicated by the Author. }
\end{gathered}
$$


Alsorption of a Beam of Heterogeneous Röntgen Rays. 781. where

$$
\xi=\epsilon^{\lambda_{1} \theta}, \quad \eta=\epsilon^{\lambda_{2} \theta}, \quad \zeta=\epsilon^{\lambda_{3} \theta}, \ldots
$$

Eliminating $A_{1} \ldots A_{n}$ from the first $(n+1)$ equations, we have

$$
\left|\begin{array}{cccc}
y_{0}, & 1, & 1, & 1 \\
y_{1}, & \xi, & \eta, & \zeta \ldots \\
y_{2}, & \xi^{2}, & \eta^{2}, & \zeta^{2} \ldots \\
& \ldots & \ldots & \ldots \\
y_{n}, & \xi^{n}, & \eta^{n}, & \zeta^{n} \ldots
\end{array}\right|=0 .
$$

Expanding the determinant, we get

$$
y_{0} \mathrm{D}_{0}+y_{1} \mathrm{D}_{1}+y_{2} \mathrm{D}_{2}+\ldots=0, \quad \text {. . . . }
$$

where $D_{s}$ is the minor corresponding to $y_{s}$, and is equal to

$$
\pm\left|\begin{array}{ccc}
1, & 1, & 1 \\
\xi, & \eta, & \zeta \\
\ldots & \ldots & \\
\xi^{n}, & \eta^{n}, & \zeta^{n}
\end{array}\right|
$$

the row $\xi^{s}, \eta^{s}, \zeta^{s} \ldots$ being left out.

We easily see that

$$
\mathrm{D}_{s}=\mathrm{P} \cdot \mathrm{S}_{s}
$$

where $\mathrm{P}$ is the product of all the differences of $\xi, \eta, \zeta, i . e$.

$$
\begin{array}{r}
(\xi-\eta)(\xi-\zeta)(\xi-\epsilon) \\
(\eta-\zeta)(\eta-\epsilon) \\
(\zeta-\epsilon) \\
(\zeta)
\end{array}
$$

and $S$ the sum of the products $\xi, \eta, \zeta$ taken $n-s$ at a time.

Thus

$$
\mathrm{S}_{0}=\xi \eta \zeta \epsilon, \quad \mathrm{S}_{1}=\xi \eta \zeta+\xi \eta \epsilon+\ldots
$$

Since $\mathrm{P}$ is a common factor of the $\mathrm{D}$ 's, we may write equation (1) as

$$
y_{0} \mathrm{~S}_{0}-y_{1} \mathrm{~S}_{1}+y_{2} \mathrm{~s}_{2} \ldots=0 \text {. }
$$

By eliminating $A_{1}, A_{2}, \ldots A_{n}$ from the $n$ equations beginning with $y_{1}$ instead of $y_{0}$, we get

$$
\begin{aligned}
& y_{1} \mathrm{~S}_{0}-y_{2} \mathrm{~S}_{1}+y_{3} \mathrm{~S}_{2} \ldots=0 ; \\
& \text { and similarly } \\
& y_{2} \mathrm{~S}_{0}-y_{3} \mathrm{~S}_{1}+y_{4} \mathrm{~S}_{2} \ldots=0 \text {, } \\
& \text { down to } \\
& y_{n-1} \mathbf{S}_{0}-y_{n} \mathrm{~S}_{1}+y_{n+1} \mathbf{S}_{2} \ldots=0 \text {. }
\end{aligned}
$$


But if $x$ is equal to any of the quantities $\xi, \eta, \zeta$, \&c.,

$$
\mathrm{S}_{0}-x \mathrm{~S}_{1}+x^{2} \mathrm{~S}_{2} \ldots=0 \text {. }
$$

Hence, eliminating $S_{0}, S_{1}, \ldots S_{n}$ from the $n+1$ equations we get

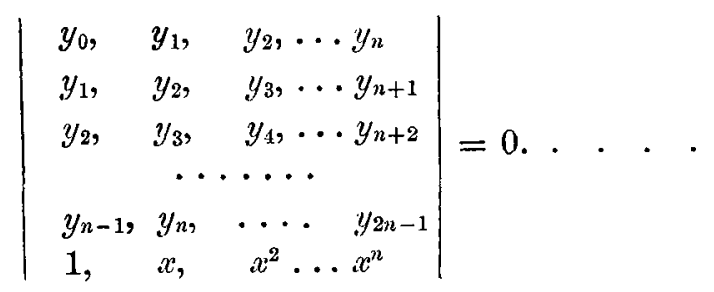

Thus the $n$ roots of this equation for $x$ are the values of $\xi, \eta, \zeta-i$. e., of $e^{\lambda_{1} \theta}, \epsilon^{\lambda_{2} \theta}$. Hence this equation determines the values of $\epsilon^{\lambda_{1} \theta}, \epsilon^{\lambda_{2} \theta}$; and since $\theta$ is lnnown, the values of the $\lambda$ 's can be immediately determined.

We can find the values of the coefficients $A_{1}, A_{2}, A_{3}$, \&c. as follows :-

From the first $n$ equations of the system $a$ we get

$$
\begin{aligned}
& \mathrm{A}_{1}=\left|\begin{array}{ccc}
y_{0}, & 1, & 1 \\
y_{1}, & \eta, & \zeta \\
\ldots & \ldots & \\
y_{n-1}, & \eta^{n-1}, & \zeta^{n-1} \\
\hline 1, & 1, & 1 \\
\xi, & \eta, & \zeta \\
\xi^{2}, & \eta^{2}, & \zeta^{2} \\
\ldots & \ldots \ldots & \\
\xi^{n-1}, & \eta^{n-1}, & \zeta^{n-1}
\end{array}\right| \\
& =-\frac{y_{0} \mathbf{s}_{n-1}-y_{1} \mathbf{s}_{n-2}+y_{2} \mathrm{~s}_{n-3} \ldots}{(\xi-\eta)(\xi-\zeta)(\xi-\epsilon) \ldots}, \quad . \quad .
\end{aligned}
$$

where $\mathrm{S}_{r}$ is the sum of the products of the $(n-1)$ quantities $\eta, \zeta, \epsilon, \ldots$ taken $r$ at a time. Thus

$$
\mathrm{S}_{n-1}=\eta \zeta \epsilon, \quad \mathrm{S}_{1}=\eta+\zeta+\epsilon+\ldots
$$

The value of a coefficient can be expressed in terms of the root corresponding to the coefficient, and does not require a knowledge of the other roots. For $\eta, \zeta, \epsilon$ are the roots of the equation

$$
x^{n-1}-\mathrm{S}_{1} x^{n-2}+\mathrm{S}_{2} x^{n-3}-\ldots=0 ;
$$

so that the polynomial is equal to

$$
(x-\eta)(x-\zeta)(x-\epsilon) \ldots
$$


of a Beam of Heterogeneous Röntgen Rays.

Writing equation (1) in the form

$$
x^{n}-p_{1} x^{n-1}+p_{2} x^{n-2}-\ldots=0,
$$

since the roots are $\xi, \eta, \zeta, \ldots$, the polynomial is equal to

Hence

$$
(x-\xi)(x-\eta)(x-\zeta) \ldots
$$

$$
\begin{aligned}
& (x-\xi)\left(x^{n-1}-\mathbb{S}_{1} x^{n-2}+\mathbb{S}_{2} x^{n-3} \ldots\right) \\
& \quad=x^{n}-p_{1} x^{n-1}+p_{2} x^{n-2}-p_{3} x^{n-3},
\end{aligned}
$$

and equating coefficients we get

$$
\begin{gathered}
\mathrm{S}_{1}+\xi=p_{1}, \\
\mathrm{~S}_{2}+\xi \mathrm{S}_{1}=p_{2}, \\
\mathrm{~S}_{3}+\xi \mathrm{S}_{2}=p_{3}, \\
\ldots \mathrm{S}_{n-1}=p_{n} .
\end{gathered}
$$

Thus all the S's can be found in terms of $\xi$.

Differentiating the identity

$$
x^{n}-p_{1} x^{n-1}+p_{2} x^{n-2}=(x-\xi)(x-\eta)(x-\zeta) \ldots,
$$

and putting $x=\xi$ after differentiation we find

$$
\begin{aligned}
n \xi^{n-1}-(n-1) p_{1} \xi^{n-2} & +(n-2) p_{2} \xi^{n-3}-\ldots \\
& =(\xi-\eta)(\xi-\zeta)(\xi-\epsilon) \ldots
\end{aligned}
$$

Thus both the numerator and denominator of (2) can be expressed in terms of $\xi$ without knowing $\eta, \zeta, \ldots$

If two roots are equal, say $\xi$ and $\eta$, the expressions for both $A_{1}$ and $A_{2}$ become indefinite. $A_{1}+A_{2}$, the quantity we require, has, however, the definite value

where

$$
-\frac{\left.y_{0} q_{n-2}-y_{1} q_{n-3}+y_{2} q_{n-4} \cdots\right)}{(\xi-\zeta)(\xi-\epsilon) \cdots}
$$

$$
\begin{aligned}
& q_{1}+\xi=\mathrm{S}_{1}, \\
& q_{2}+\xi q_{1}=\mathrm{S}_{2}, \\
& q_{3}+\xi q_{2}=\mathrm{S}_{3},
\end{aligned}
$$

where the S's are the same as in equation (3). The denominator is

$$
(n-1) \xi^{n-2}-(n-2) \mathrm{S}_{1} \xi^{n-3}+(n-3) \mathrm{S}_{2} \xi^{n-4} \ldots
$$

Mr. R. A. Herman of Trinity College, Cambridge, has pointed out to me that the determinant in equation (2) is of the type discussed by Sylvester in a paper in the Philosophical Magazine, Nov. 1851, and ealled by him a canonizant. 